\title{
Futuros de la inteligencia artificial
}

\author{
Guadalupe Vadillo Bueno
}

\section{Resumen}

Dado el desarrollo acelerado de la inteligencia artificial y su impacto en una inmensa gama de actividades y disciplinas, existe un interés por imaginar sus posibles escenarios en el futuro. Se presenta un compendio de opiniones de cuatro expertos mexicanos que se complementa con una síntesis de algunos estudios previos. En especial se abordan la singularidad, las oportunidades que la inteligencia artificial ofrece y los riesgos que puede suponer.

Palabras clave: Inteligencia artificial, futuro, estudios prospectivos.

\section{FUTURES OF ARTIFICIAL INTELLIGENCE}

\section{Abstract}

Due to the fast evolution of artificial intelligence and its impact on a variety of activities and disciplines, there is interest in imagining its possible future scenarios. We present a summary of contributions from four Mexican researchers, complemented with some previous studies and insights on the subject. They focus on the singularity, the opportunities artificial intelligence entails as well as the risks that it may involve.

Keywords: artificial intelligence, future, future studies. 


\section{Guadalupe Vadillo Bueno}

Directora del Bachillerato a Distancia (B@unam), Universidad Nacional Autónoma de México (UnAm). Psicóloga, con maestría en Educación del sobresaliente y Doctorado en Educación. Cuenta con entrenamientos en Terapia breve y junguiana. Fue Coordinadora de psicología, Asesora de vicerectorías y Directora de educación continua en la Universidad de las Américas y por 12 años supervisó su Centro de idiomas. Ha sido asesora de 37 tesis de licenciatura, tres de maestría y tres doctorales. Asesora para la innovación educativa por seis años del Colegio Hebreo Tarbut. Durante un año, tuvo el cargo honorario de Directora ejecutiva del Espacio Común de Educación Superior a Distancia. Actualmente es Coordinadora de la Red de Bachilleratos Universitarios Públicos a Distancia. Ha diseñado, asesorado, tutorado y coordinado diversos cursos y programas en línea. Es autora de dos de los primeros cursos abiertos masivos en línea (mooc) en México, a través de unAM - Coursera: Ser más creativos con más de 340 mil estudiantes registrados y Aprender, con más de 65 mil. Desde 2011 es docente en línea de la carrera de Psicología de la FES-Iztacala unAM. 
No hacer daño a un ser humano, obedecer las órdenes dadas por humanos (excepto si entran en conflicto con la primera ley) y proteger su propia existencia (excepto si la protección entra en conflicto con las dos primeras leyes).

2"After "Runaround" appeared in the March 1942 issue of Astounding, I never stopped thinking about how minds might work. Surely we'd someday build robots that think. But how would they think and about what? Surely logic might work for some purposes, but not for others. And how to build robots with common sense, intuition, consciousness and emotion? How, for that matter, do brains do those things?" (Markoff, 1992, versión digital sin página).
"Somos los guardianes del futuro de la vida ahora, mientras moldeamos la era de la inteligencia artificial".

Max Tegmark

\section{El interés por el futuro}

Viajemos hacia atrás en el tiempo, 77 años, cuando Asimov publicó un cuento titulado "Runaround", donde introducía las tres leyes de la robótica" (Kaminka, Sponkoini-Stern, Amir, Agmon y Bachelet, 2017). Unos años más tarde, en 1950, Turing publicó el artículo que pasaría a la historia: "Computing machinery and intelligence" en el que se preguntaba si las máquinas podían pensar (Castelfranchi, 2013). Finalmente, en 1956, Minsky y McCarthy acuñaron el término de inteligencia artificial durante el Dartmouth Summer Research Project on Artificial Intelligence (Howard, 2019). Minsky contaría al New York Times que después de leer "Runaround", en marzo de 1942, nunca dejó de pensar cómo las mentes pueden trabajar y que algún día se construirían robots que piensen.²

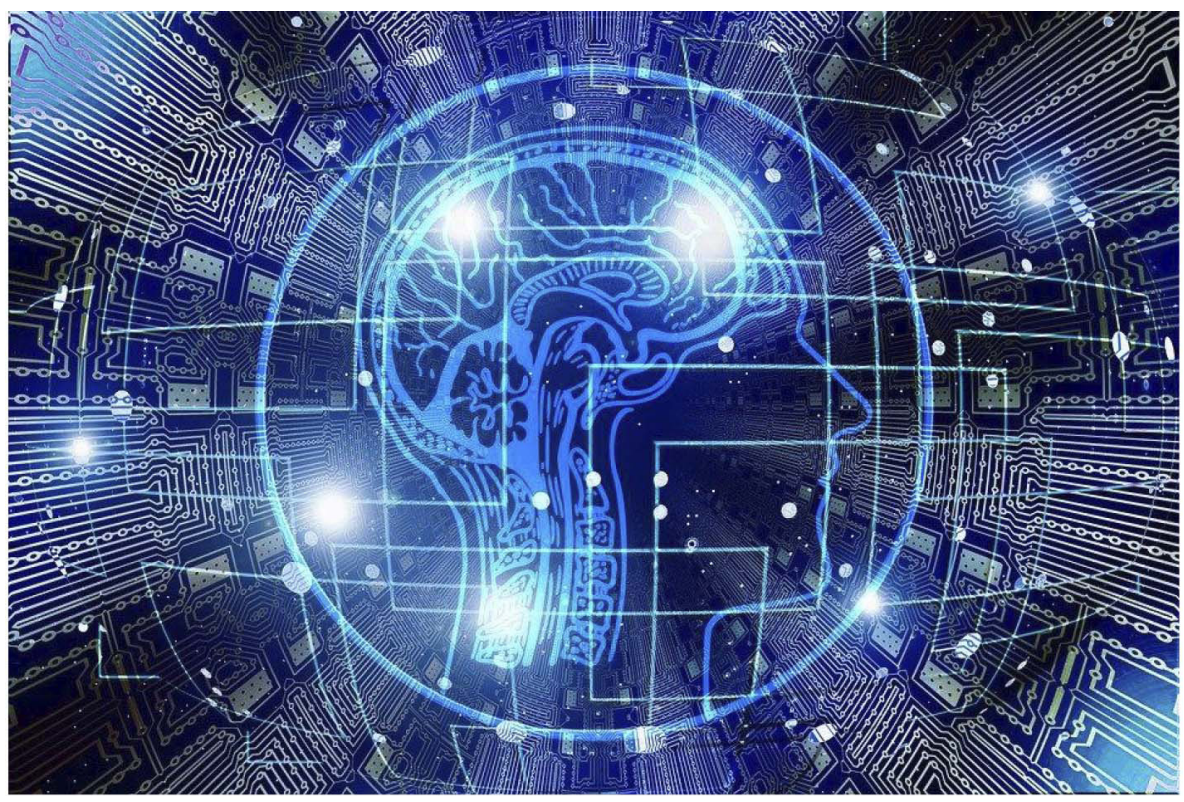

Hoy, la ia se desarrolla constantemente a través del esfuerzos de científicos y tecnólogos en muchos países del mundo. El trabajo e interés por el tema se puede evidenciar con la cantidad de resultados que se obtienen al buscar, en Google Scholar, el término en inglés: japarecen más de tres millones de entradas! Una gran cantidad de líneas de investigación y desarrollo están abiertas, con aplicaciones en prácticamente todos los campos de la actividad humana. Resulta tan vertiginoso el trabajo que se hace y son tan impresionantes los adelantos que se aportan, que muchos especialistas se han dedicado a investigar y a pensar sobre el futuro de la IA. 
En este artículo presentamos las opiniones de cuatro expertos mexicanos a quienes consultamos y las complementamos con conclusiones reportadas en algunos estudios prospectivos y reflexiones recientes. Esta información conjunta intenta responder a la pregunta: ¿qué nos depara en el futuro la IA?

\section{Nuestro oráculo especial}

Para responder a esta pregunta convocamos a cuatro expertos en el tema que accedieron a constituirse en un oráculo para este artículo. Los presentamos en la imagen 1.
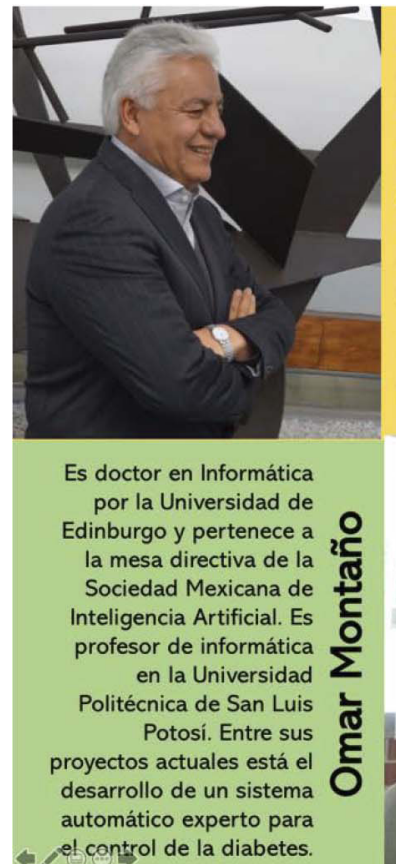

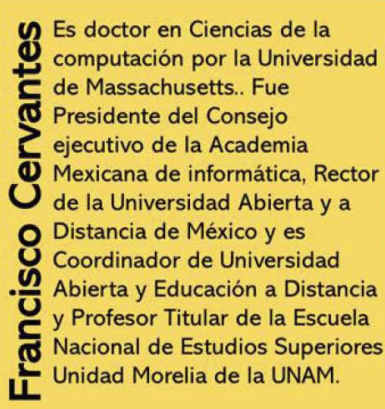
L.

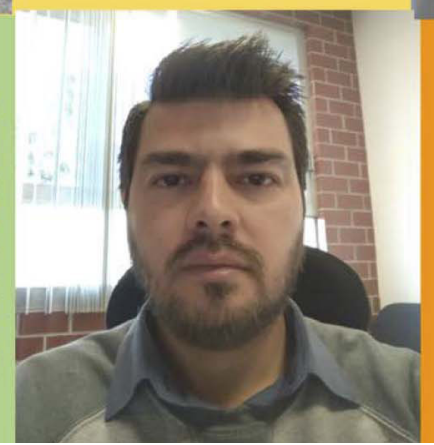

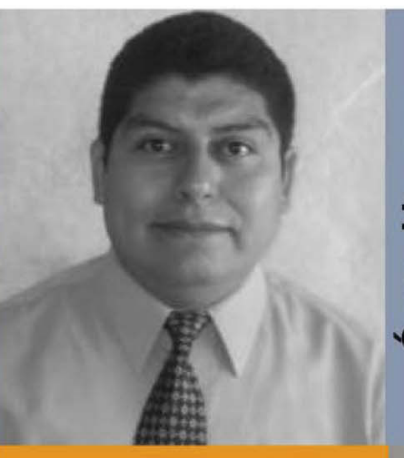

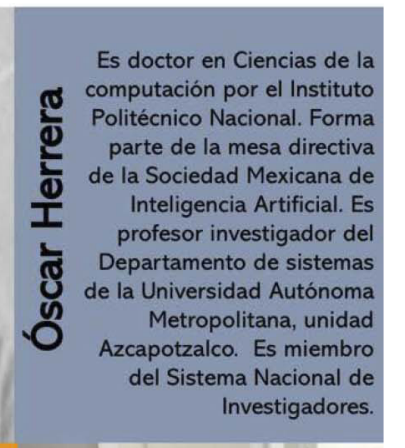

Es doctor en Diseño y control de sistemas autoorganizantes por la Universidad Libre de Bruselas y tiene un postdoctorado en el Instituto de Sistemas Complejos de Nueva Inglaterra. Es investigador de la UNAM, titular del Laboratorio de sistemas auto-orgnizantes.

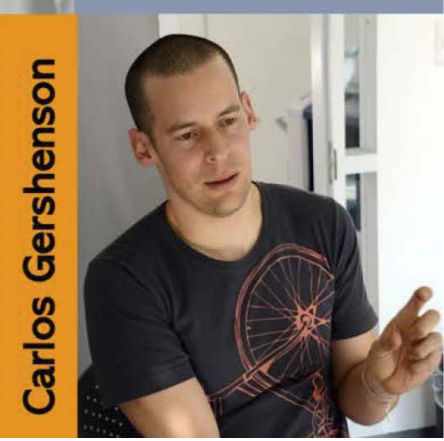

Imagen 1. Expertos que colaboraron en este estudio (fotos aportadas por los especialistas).

\section{¿Qué indican los estudios de futuro sobre la IA?}

Cuando exploramos el campo, tenemos un abanico que va desde la cautela extrema y predicciones sobre la imposibilidad de que la IA compita con la inteligencia humana, hasta una seguridad total acerca de su potencial espectacular. Por un lado, Steven Livingston, de la George Washington University, y Mathias Risse, de Harvard, (2019) destacan que no existe aún una "superinteligencia", o inteligencia artificial general, es decir, un algoritmo o una serie de algoritmos que al menos tengan igual capacidad que los humanos en diferentes dominios. Por otra parte, Anthony Miller (2019), desde Gran Bretaña, considera que la humanidad debe decidir subirse a la emocionante ola de cambios que trae consigo la IA o resistirse al cambio inevitable que tendrá el mundo como lo conocemos. Concluye que, si la humanidad no se decide por la colaboración, seremos ciudadanos de segundo nivel en nuestro propio mundo. 
Demos una mirada a lo que se ha logrado hasta ahora, para poder medir los alcances de las predicciones. Livingston y Risse (2019), señalan que los intentos actuales de expandir la adaptabilidad de algoritmos a distintos dominios de problemas, están representados por DeepMind de Google y por Google Brain. Del primero se desprende el programa de cómputo AlphaGo quizá la evidencia más clara del desarrollo en este sentido; este programa se desarrolló para jugar ese juego de mesa. En su entrenamiento inicial se usaron treinta millones de jugadas de 160 mil partidas de excelentes jugadores de ajedrez como input. Su "aprendizaje" posterior fue por reforzamiento, jugando contra versiones previas de sí mismo durante miles de veces, con lo que hay mejoras en cada iteración. La primera iteración se Ilamó AlphaGo Zero y en las primeras tres horas, logró la competencia de un humano amateur, en 19 horas las de uno avanzado y en tres días venció a la versión AlphaGo que había superado en 2016 al mejor jugador profesional de Go del mundo.

Los investigadores orientan su escrito a ia y derechos humanos, y señalan que ya existen incidencias en este campo en la actualidad. Por ejemplo, se logró la captura de un jefe militar libio a partir de imágenes de ejecuciones que él ordenó o realizó, y que aportaban indicios de lugares geográficos en tiempos específicos, que después se contrastaron con imágenes satelitales. Asimismo, Livingston y Risse señalan que en antropología forense es posible contar con el apoyo de IA para secuenciar ADN en forma paralela y masiva, con objeto de establecer la identidad de las víctimas con base en unos cuantos restos óseos. Si bien ha habido aportaciones importantes de la $\mathbf{I A}$, tenemos que tomar en consideración que puede haber errores en los procesos. Un ejemplo es el caso de una IA que, en lugar de aprender a distinguir tanques de guerra, aprendió a distinguir los paisajes donde se encuentran (Yudkowsky, 2008 en Yampolskiy, 2019).

\section{Expertos y no expertos en la creación de futuros de la IA}

Carlos Gershenson, uno de los especialistas que consultamos, considera que la relación entre los humanos y la IA, con el tiempo, se volverá más codependiente y que la tendencia es hacia una simbiosis, aunque los cambios en la siguiente década no serán notorios. Asimismo, señala que la ı estará más embebida en nuestra tecnología. Óscar Herrera piensa que la IA dentro de unos años pasará desapercibida, como sucede con la luz o el Internet. Imagina que la ia estará inmersa en cada dispositivo, tecnología, medio de difusión, modelo educativo, ideológico y formativo, además de ser parte de los modelos de negocio. Omar Montaño considera que los asistentes personales que tendremos serán más eficientes que los humanos y que la ia tendrá un impacto importante en medicina, ciberseguridad y desarrollo de software libre de errores, entre otras áreas. Por su parte, Francisco Cervantes enfatiza el uso de aplicaciones basadas en ia para actividades como el diagnóstico médico y de uso cotidiano, como los carros autónomos, y los audífonos y lentes enriquecidos con realidad aumentada o virtual. 
¿Y qué dicen los no expertos? La Fundación Mozilla (2019) publicó en noviembre de 2019 los hallazgos de la encuesta a 66,800 personas de todo el mundo sobre este tema. Los resultados principales fueron:

- 86.8\% contestó que no sabía mucho sobre ia o tenía algunas nociones,

- 80\% de los jóvenes de 19 a 24 años considera tener más conocimientos que los de 24 a 44 años (75\%) o los de 45 a 75 (59\%);

- en cuanto a los sentimientos que la ı genera, el 32\% de la gente señala preocupación, el 30\% curiosidad y el 27\% ilusión; y

- la mayor parte considera que la ia debe ser mucho más inteligente que los seres humanos y sólo 10\% que es mejor que sea tonta.

Como vemos, hay mucho que explorar en este tema. Para adentrarnos en él, demos un vistazo a tres rubros específicos: la singularidad, las oportunidades y los retos de la IA.

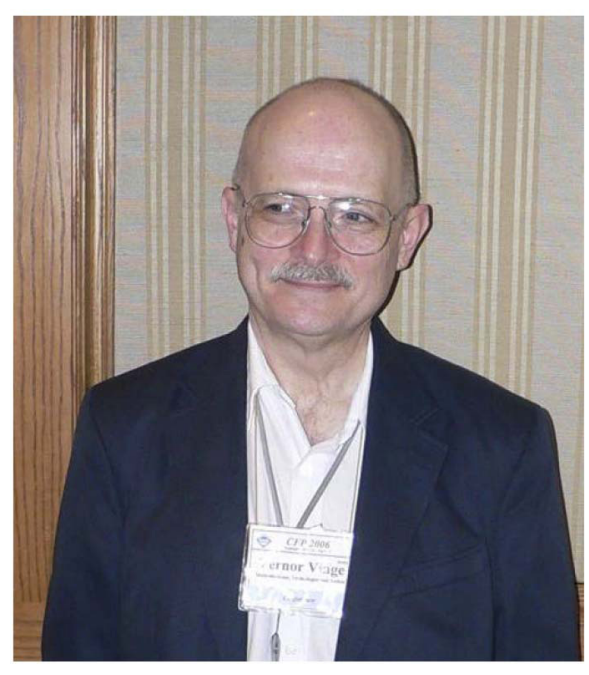

\section{Singularidad}

Vernor Vinge, un profesor de matemáticas y escritor de ciencia ficción, quien popularizó el término singularidad que se refiere a un punto hipotético en el futuro, cuando la inteligencia artificial supera a la del hombre. Se trata del posible momento en el que el desarrollo tecnológico con ciclos de auto-mejoramiento lleva a que el agente inteligente no humano sobrepase a la inteligencia humana. Vinge señaló en su ensayo de 1993 "The comming technological singularity" que se sorprendería si sucediera antes de 2005 o después de 2030 (en Zovko, 2018).

Hay poco acuerdo en cuanto a si ese momento hipotético llegará o no. Los expertos mexicanos que consultamos son un ejemplo de estas diferencias: Carlos Gershenson, indicó que nunca llegará, que se trata de un concepto equivocado y que una tendencia exponencial no lleva a una singularidad, tendría que tratarse de una función hiperbólica. Además, piensa, que las tendencias tienden a cambiar: la Ley de Moore lleva años desacelerándose y aunque nuestro poder de cómputo se ha incrementado exponencialmente durante décadas, nuestra habilidad para desarrollar software lo ha hecho de manera sublineal. Por su parte, Omar Montaño indicó que sí sucederá dentro de 20 o 30 años, mientras que Óscar Herrera señaló que no se notará y Francisco Cervantes que ya llegó, en tanto que la adaptabilidad humana es menor al crecimiento tecnológico, lo que nos obliga a aprender más rápidamente y crecer de forma inteligente. 
Para hacer evidente la disparidad de posiciones, basta con leer el número que editaron Adriana Braga, de la Pontificia Universidad Católica de Río de Janeiro y Robert Logan, de la Universidad de Toronto (2019). En este trabajo llevaron a cabo una convocatoria de artículos para la revista Information y se presentaron textos a favor y en contra de la idea de la singularidad. Los críticos señalan que la inteligencia humana no sólo incluye operaciones lógicas y computación, sino también deseos, juicios, experiencias, curiosidad, imaginación y humor, entre otros elementos. Concluyen que lo mejor es la colaboración entre humanos e IA, que ésta por sí sola nunca reemplazará la inteligencia humana y que no se puede confiar en la ia para obtener la respuesta correcta.

Miller (2019) está seguro de que la singularidad llegará y se sobrepasará. Debido a ello, opina que los humanos y la iA se esforzarán por llegar a la inteligencia artificial general, lo que significa una evolución conjunta de humanos e IA. Considera que son tres los factores que se entrelazarán para esa transformación: Big data, la computación cuántica y la tecnología de interfaz cerebro-computador, que pueden llevar a la ıA a una velocidad de evolución casi inimaginable.

Veamos uno por uno lo que estos factores suponen.

1. Big data implica:

- grandes cantidades de datos generados por fuentes diversas, como las comunicaciones, la manufactura o las áreas de salud, pero también la interacción humano-ı y el internet de las cosas,

- una amplia variedad en que los datos pueden usarse para analizar un evento o un predicamento, y

- una velocidad creciente de los datos, ya que con el desarrollo de la tecnología crece la cantidad de datos.

2. La computación cuántica: tiene la meta de lograr millones de veces más poder que el que ofrecen las computadoras clásicas. En lugar de trabajar con ceros y unos, la computación cuántica usa cúbits, que, a través de la llamada superposición, en un mismo momento pueden ser cero, uno y otros estados. Al combinarse los cúbits, el número de estados crece exponencialmente, con lo que la velocidad de las computadoras cuánticas crece a pasos agigantados. Se espera que llegue pronto la "supremacía cuántica", momento en que la computación cuántica supere a la clásica.

3. Por otra parte, la interfaz cerebro-computadora: permite a los usuarios el control de dispositivos externos a partir de señales cerebrales. Aunque está en una fase muy inicial de desarrollo, resulta prometedora.

Lo comentado sobre la singularidad nos deja en una posición poco precisa: incluso grandes estudiosos de la IA no acuerdan si llegará o no. Sin embargo, lo que ya está aquí es una amplia gama de oportunidades de usar la is en muy 
distintos campos de actividad humana. En esta sección exploraremos apenas algunas de estas oportunidades.

\section{Oportunidades}

Tanto Francisco Cervantes como Omar Montaño destacan las aportaciones que la IA puede hacer en el área médica. Shah (2019) coincide con ellos: señala que para 2030 los médicos tendrán asistentes digitales que escucharán las consultas, tomarán notas sobre el cuidado del paciente, y cuestiones de contabilidad. Gestionarán canalizaciones y arreglarán cuidados preventivos basados en evidencia. Sin embargo, el médico seguirá siendo importante.

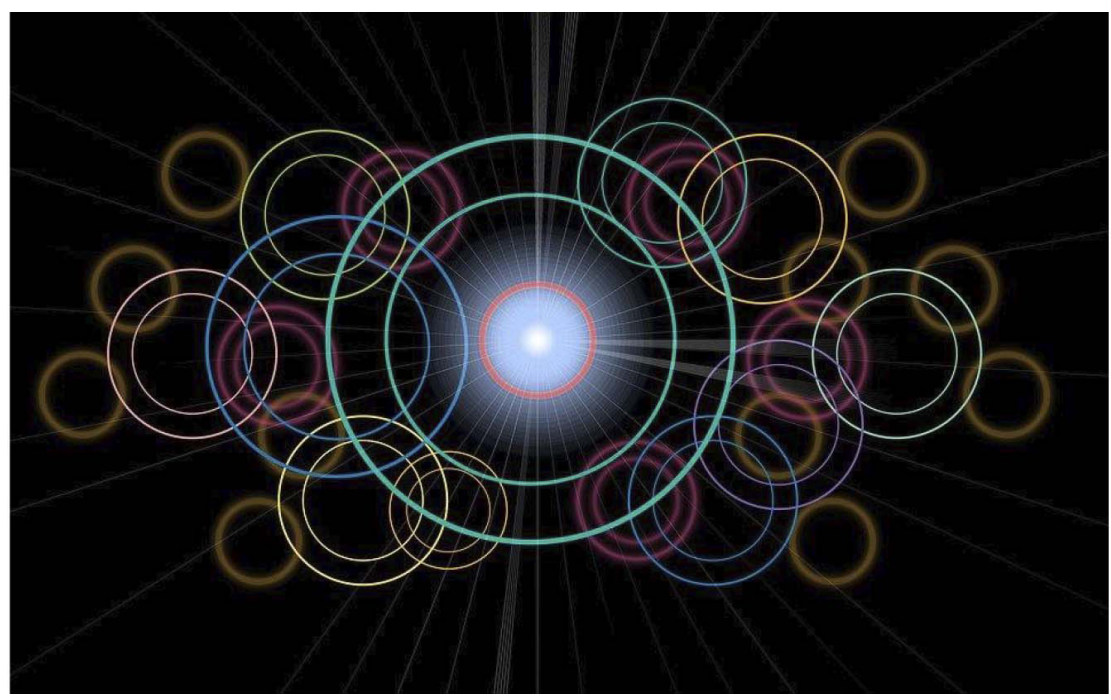

Omar Montaño imagina que programas de computadoras crearán programas (compiladores, sistemas operativos, algoritmos o rutinas en sistemas críticos) libres de errores; mientras Francisco Cervantes considera que un problema no resuelto será la comprensión de lenguaje natural. Óscar Herrera considera que los problemas insolutos en el presente que la ia puede ayudar a solucionar son los que requieren una amplia capacidad de cómputo, de grandes volúmenes de datos multimedios (el uso del texto desaparecerá, es decir el modelo clásico de libro como lo conocemos ahora), por lo que, ante la falta de lectura, se evolucionará a "audiolibros" por llamarlos de alguna manera, pero con nuevas tecnologías de transfusión rápida del conocimiento (una "infusión mental") que contemple emociones, en donde "el texto queda limitado y pasará a nuevos modelos de comunicación y transmisión de ideas, almacenadas en nuevos repositorios de conocimiento interactivos". Carlos Gershenson, por su parte, considera que más que resolver problemas, la IA ayudará a mejorar las soluciones actuales. Hay problemas complejos cuyas respuestas hoy no tenemos, y considera que usando técnicas de iA con grandes cantidades de datos (algunos que aún no tenemos), probablemente algunos se puedan resolver. 


\section{Riesgos}

Por supuesto, escuchar tantas bondades de la IA nos hace levantar la ceja y preguntarnos por el otro lado de la moneda, ¿cuáles son los riesgos que implica? Turchin (2019) introduce el concepto de IA peligrosa: una IA suficientemente poderosa como para crear riesgos catastróficos: puede o no ser una inteligencia artificial general y quizá no pase la prueba de Turing. Probablemente esté cerca de un nivel humano pero no tenga conciencia. Hay tres formas en que puede volverse peligrosa: podría actuar, de acuerdo con Turchin, en el medio libre como Internet y desempeñarse mejor que un humano en tareas humanas, debido quizá a su habilidad para crear un modelo del mundo y su capacidad para procesar lenguaje natural. La segunda forma es que facilite investigación peligrosa en campos como la biotecnología. La tercera, y que genera mayor preocupación, es si una IA inicia un desarrollo explosivo de inteligencia a través de un proceso de rápido auto-mejoramiento.

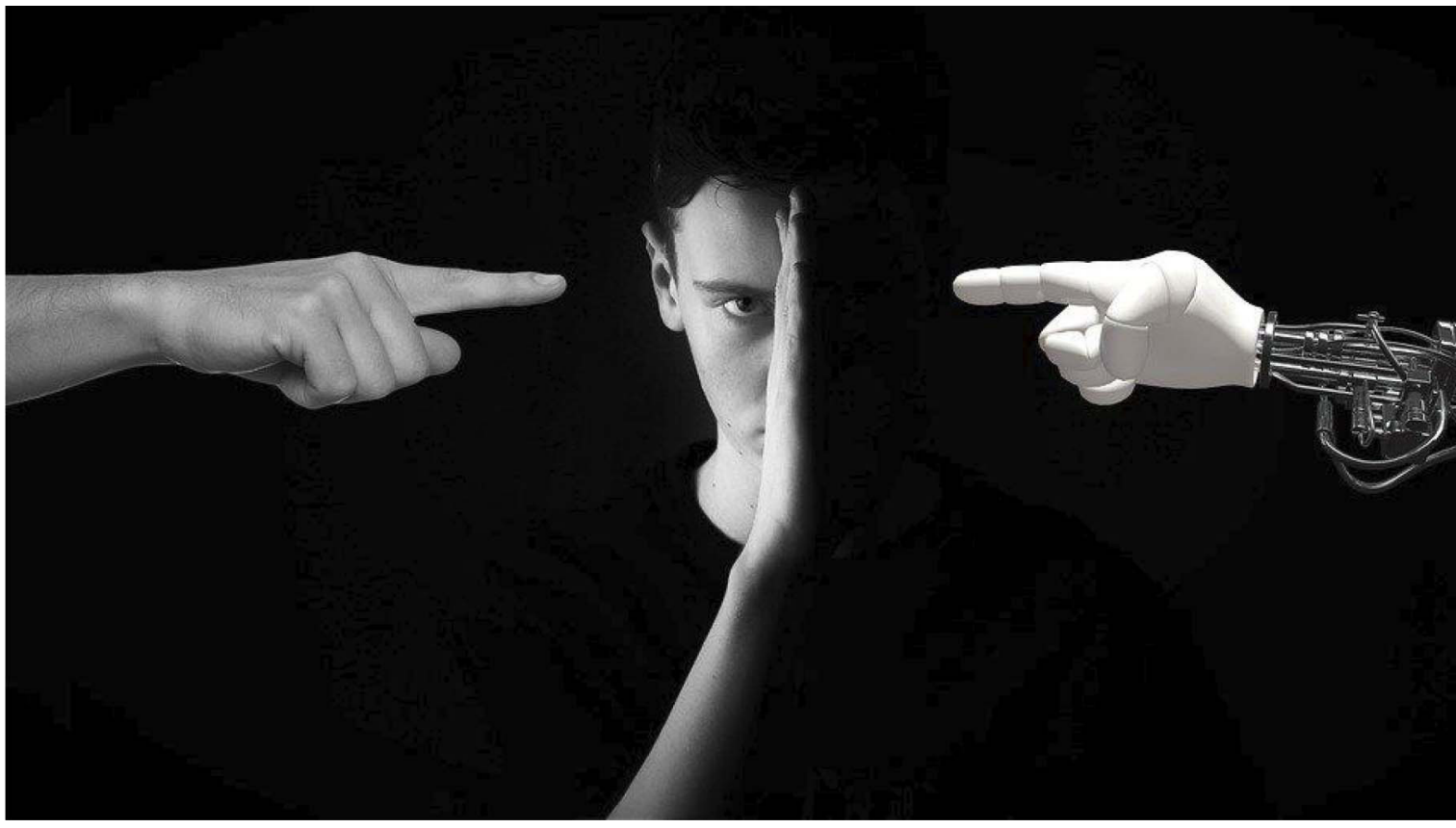

Óscar Herrera considera que, al igual que muchas otras tecnologías como la radio - la televisión que se pensaron como vehículos para acercar el conocimiento a entidades remotas, o Internet que se pensó podría aportar una mayor educación, podrían terminar siendo medios de manipulación de las masas. Por ejemplo, se puede lograr un mayor nivel de control al tener acceso a datos demasiado precisos e invasivos a partir del uso de sensores para que nadie esté fuera de alcance. Concluye preguntándose: ¿cuándo se ha visto que las nuevas tecnologías proporcionen una mejor calidad de vida como eje central?

Los editores del número especial sobre IA en el California Management Review (Haenlein \& Kaplan, 2019) señalan que nadie sabe los alcances de la IA: "puede ser desde que nos permitirá desarrollar nuestra inteligencia (como 
sostiene Kurzweil, de Google), hasta que nos conduzca a la tercera guerra mundial" (como teme Elon Musk). Opinan que su desarrollo implica desafíos de carácter legal, ético y filosófico.

Al consultar con nuestro oráculo de expertos mexicanos, vemos un acuerdo total. Señalan que hay un riesgo potencial que se deriva no de ella misma, sino de los usos que la gente haga a partir de sus posibilidades. Como dice Carlos Gershenson: se puede usar para beneficiar a las personas o para dominarlas, o como señala Francisco Cervantes, para destruirlas. Óscar Herrera destaca que los riesgos que la ia plantea son los mismos que los de cualquier otra tecnología con inspiración bélica. Omar Montaño propone que se generen organismos reguladores que observen y auditen estas tecnologías.

\section{Conclusiones}

"La verdadera generosidad hacia el futuro reside en darlo todo al presente".

Albert Camus

Esta visita a las reflexiones de especialistas sobre posibles futuros de la inteligencia artificial nos deja pensando que tenemos una responsabilidad sobre esos escenarios. Las decisiones pequeñas y grandes que tomamos, las posturas que asumimos y la presión o la omisión que hacemos pueden definir las trayectorias en que se desenvuelva la vida de nuestros descendientes.

Por lo pronto, la IA ya es parte de nuestra vida: cuando hablamos con un agente no humano al consultar nuestro saldo en el banco, cuando nos resuelve preguntas un chatbot en una página de internet, cuando nuestro médico se

Imagen 3. Opiniones de expertos con respecto a si ya somos o no una unidad biotecnológica. apoya en iA para darnos un diagnóstico. Entonces, ¿nos encaminamos a ser una unidad biotecnológica con ella? Tres de nuestros cuatro expertos consideran que ya llegamos a ese punto y sus opiniones se pueden ver en la imagen 3.

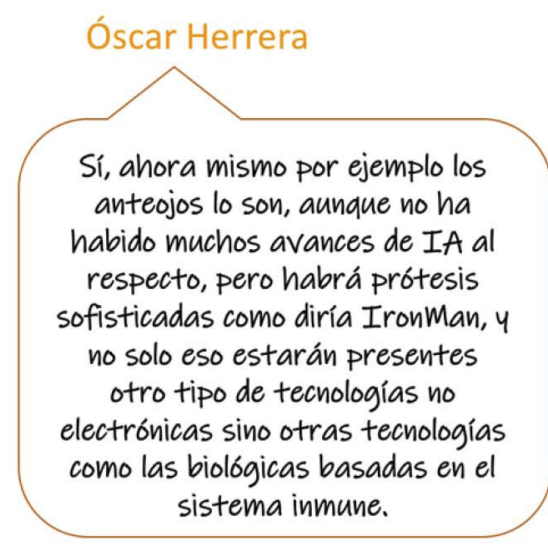

De alguna manera se podría ver que ya somos una unidad

biotecnológica. Ejemplo de esto son los

marcapasos, reguladores de insulina, prótesis, lentes de contacto o dispositivos para la audición.

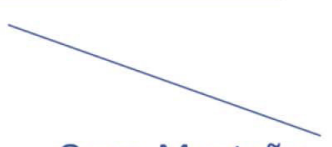

Omar Montaño

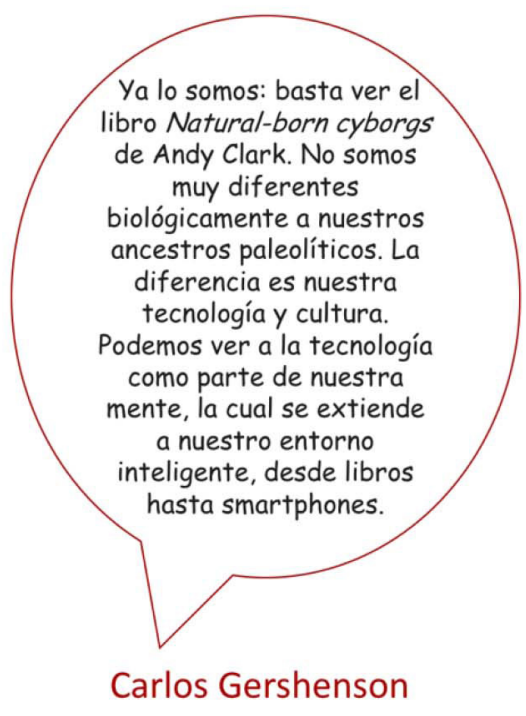

Carlos Gershenson 
Sin embargo, Francisco Cervantes señala que "más bien el humano incorporará a su cuerpo cada vez más partes de autómata".

Enfrentamos una gran incertidumbre con relación al futuro de la IA. Podemos estar ciertos de que nos acompañará en diferentes ámbitos de nuestra vida y como todo acompañante, más conviene conocerla con detalle.

\section{Referencias}

Braga, A. y Logan, R. K. (2019). Information, 10(2). Dol: https://doi.org/10.3390/ info10020073.

* Castelfranchi, C. (2013). Alan Turing's "Computing machinery and intelligence". Topoi, 32 (2), 293-299. Dol: https://doi.org/10.1007/s11245-013-9182-y .

* Haenlein, M. y Kaplan, A. (2019). A Brief History of Artificial Intelligence: On the Past, Present, and Future of Artificial Intelligence. Calif. Bus. Rev., 61(4), 5-14. Dol: https://doi.org/10.1177/0008125619864925 .

* Howard, J. (2019). Artificial intelligence: Implications for the future of work. Am. J. Ind. Med., 62, 917-926. Dol: https://doi.org/10.1002/ajim.23037.

* Kaminka, G. A., Sponkoini-Stern, R., Amir, Y., Agmon, N. y Bachelet, I. (2017). Molecular Robots Obeying Asimov's Three Laws of Robotics. Artif. Life, 23(3), 343350. Dol: https://doi.org/10.1162/ARTL a 00235.

- Livingston, S. y Risse, M. (2019). The Future Impact of Artificial Intelligence on Humans and Human Rights. Ethics and International Affairs, 33(2), 141-158. Dol: https://doi.org/10.1017/s089267941900011x.

* Markoff, J. (1992). Technology: A celebration of Isaac Asimov. The New York Times, 12 de abril. Recuperado de: https://www.nytimes.com/1992/04/12/business/ technology-a-celebration-of-isaac-asimov.html?pagewanted=all\&src=pm.

- Miller, A. (2019). The intrinsically linked future for human and Artificial Intelligence interaction. J Big Data, 6(38). Dol: https://doi.org/10.1186/s40537-019-0202-7.

* Mozilla Foundation. (2019). Preguntamos a distintas personas de todo el mundo qué piensan sobre la inteligencia artificial. Esto es lo que descubrimos. Recuperado de: https://foundation.mozilla.org/es/blog/we-asked-people-around-the-worldhow-they-feel-about-artificial-intelligence-heres-what-we-learned/.

Raul654 (2006). Vernon Vinge en 2006. Recuperada de: https://commons. wikimedia.org/wiki/File:Vernor Vinge.jpg.

- Shah, N.R. (2019). Health Care in 2030: Will Artificial Intelligence Replace Physicians? Ann Intern Med., 170(6), 407-408. Dol: https://doi.org/10.7326/M19-0344 .

* Turchin, A. (2019). Assessing the future plausibility of catastrophically dangerous IA. Futures, 107, 45-58. Dol: https://doi.org/10.1016/j.futures.2018.11.007. 
* Yampolskiy, R. V. (2019). Predicting future IA failures from historic examples. Foresight, 21(1), 138-152. Dol: https://doi.org/10.1108/FS-04-2018-0034.

Zovko, V. (2018). Management in the year 2050. INDECS, 16(3-B), 417-426. DoI: https:// doi.org/10.7906/indecs.16.3.14.

\section{Cómo citar este artículo}

* Vadillo, Guadalupe (2020). Futuros de la inteligencia artificial. Revista Digital Universitaria (RDU). Vol. 21, núm. 1 enero-febrero. Dol: http://doi.org/10.22201/ codeic.16076079e.2020.v21n1.a3.

Recepción: 27/11/2019. Aprobación: 05/12/2019 